Supporting Information

\title{
Improving the Purity and Uniformity of Pd and Pt Nanocrystals by Decoupling Growth from Nucleation in a Flow Reactor
}

Ruhui Chen, ${ }^{\dagger}$ Zhiheng Lyu, ${ }^{\dagger}$ Yifeng Shi, ${ }^{\dagger}$ and Younan $\mathrm{Xia}^{*}, \dagger,+, \$$

†School of Chemistry and Biochemistry, Georgia Institute of Technology, Atlanta, Georgia 30332, United States

\$School of Chemical and Biomolecular Engineering, Georgia Institute of Technology, Atlanta, Georgia 30332, United States

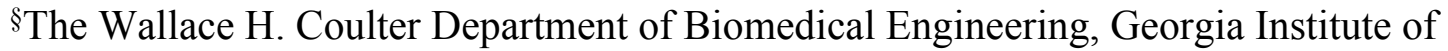
Technology and Emory University, Atlanta, Georgia 30332, United States

*Corresponding author. Email: younan.xia@bme.gatech.edu 

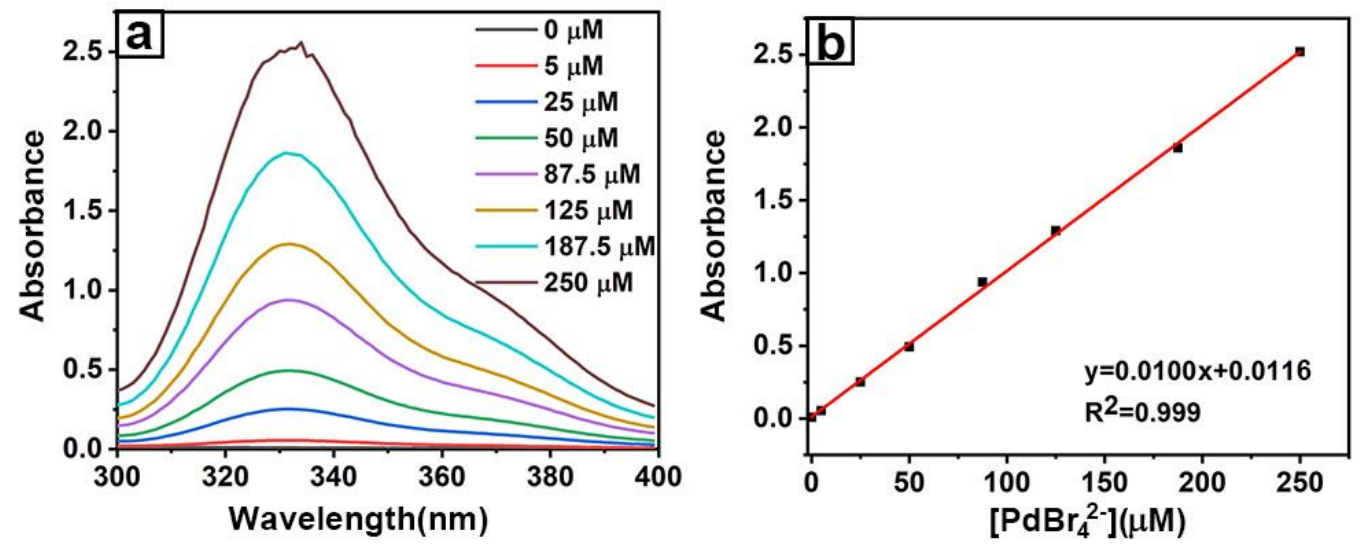

Figure S1. (a) UV-vis spectra of a set of $\mathrm{PdBr}_{4}{ }^{2-}$ solutions with different concentrations. (b) Calibration curve showing the linear correlation between the concentration of $\mathrm{PdBr}_{4}{ }^{2-}$ and the absorbance at $332 \mathrm{~nm}$. 


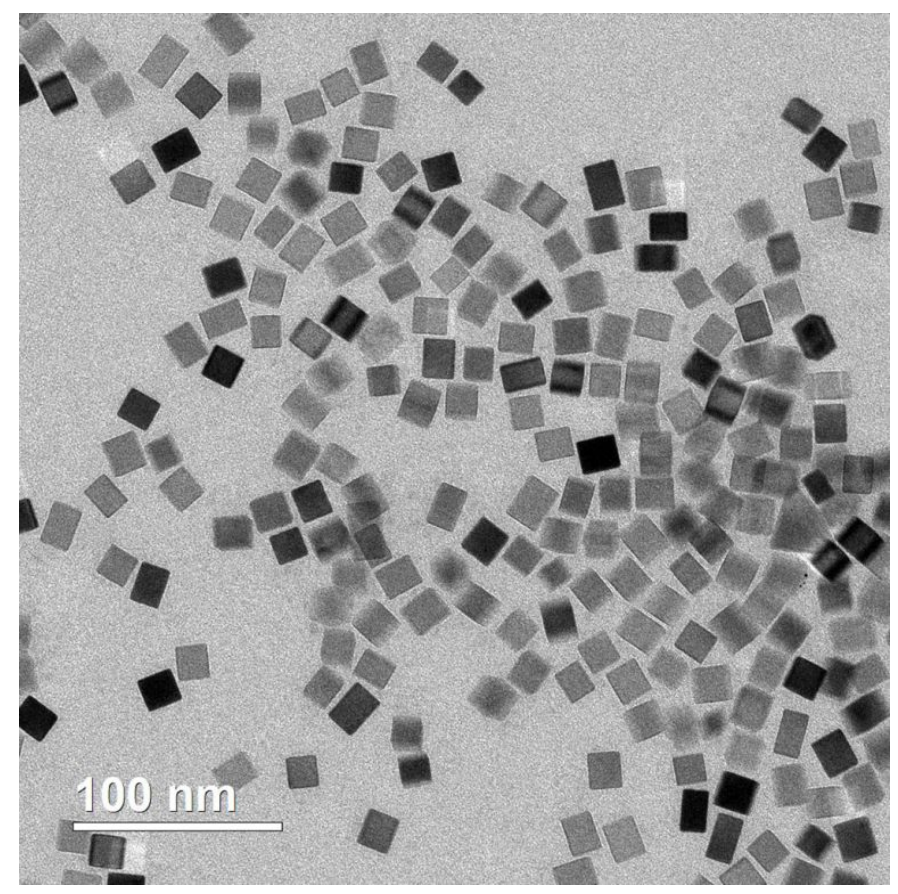

Figure S2. TEM image of Pd nanocubes synthesized by following the conventional protocol, during which $\mathrm{PdCl}_{4}{ }^{2-}$ is reduced by AA at $80{ }^{\circ} \mathrm{C}$ in an aqueous solution containing $\mathrm{KBr}$ and $\mathrm{PVP}$. 

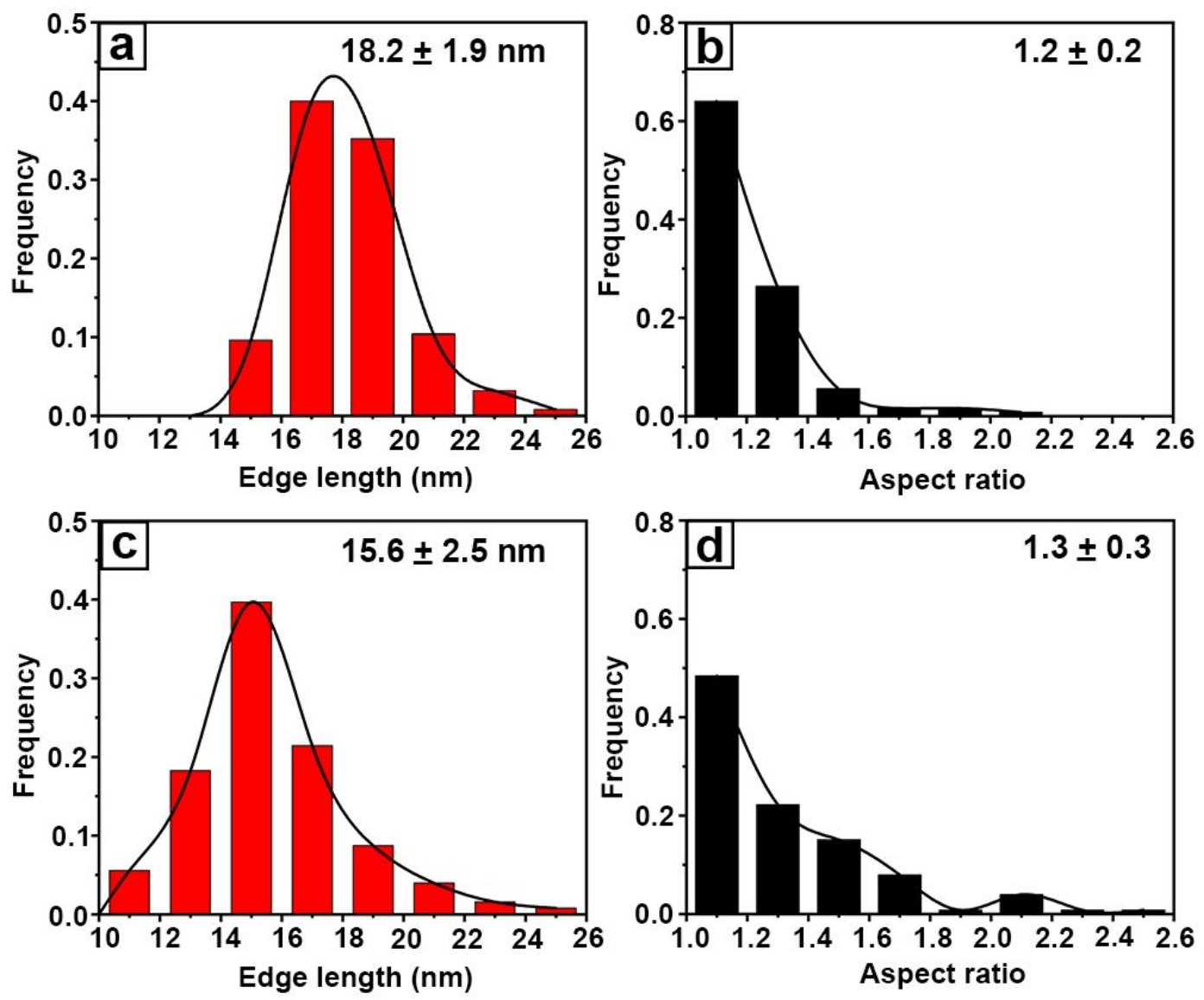

Figure S3. Histograms showing the edge length and aspect ratio distribution of Pd nanocubes synthesized by $(\mathrm{a}, \mathrm{b})$ conducting the nucleation at $95{ }^{\circ} \mathrm{C}$ for $30 \mathrm{~s}$, followed by growth at room temperature for $48 \mathrm{~h}$ (i.e., the product shown in Figure 1b), and (c, d) following the conventional protocol (i.e., the product shown in Figure S2), respectively. 


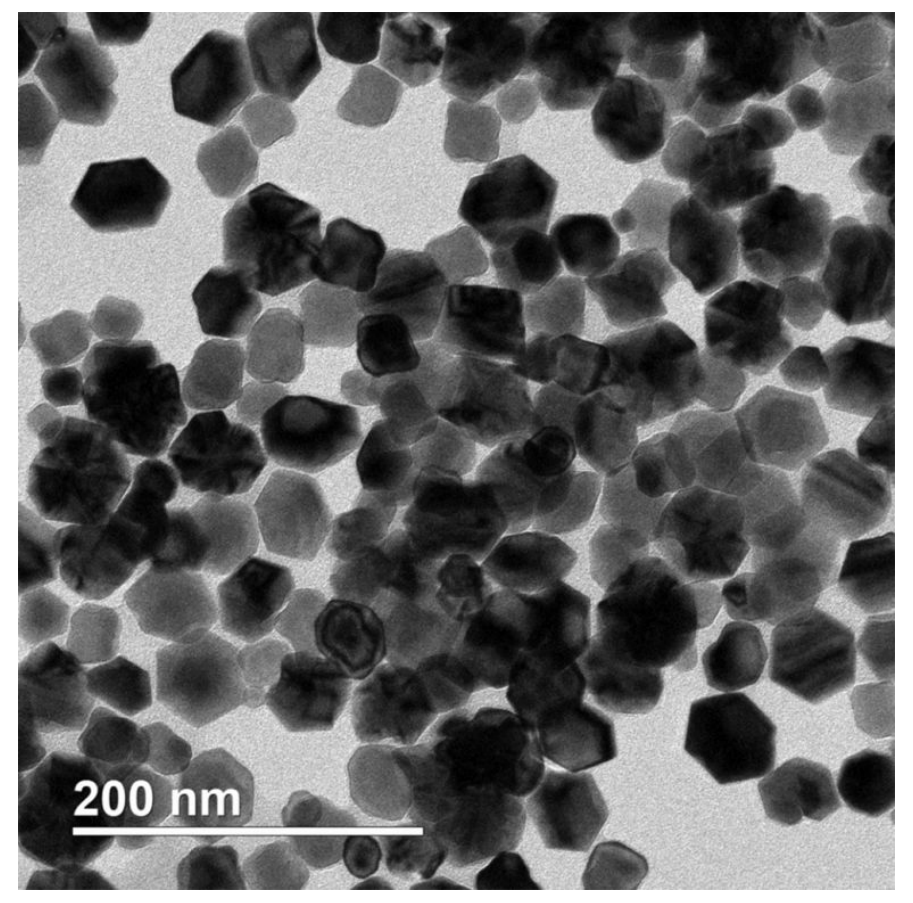

Figure S4. TEM image of Pd nanocrystals obtained using the standard protocol except that a 20 $\mathrm{mL}$ glass vial was used for the nucleation step. 


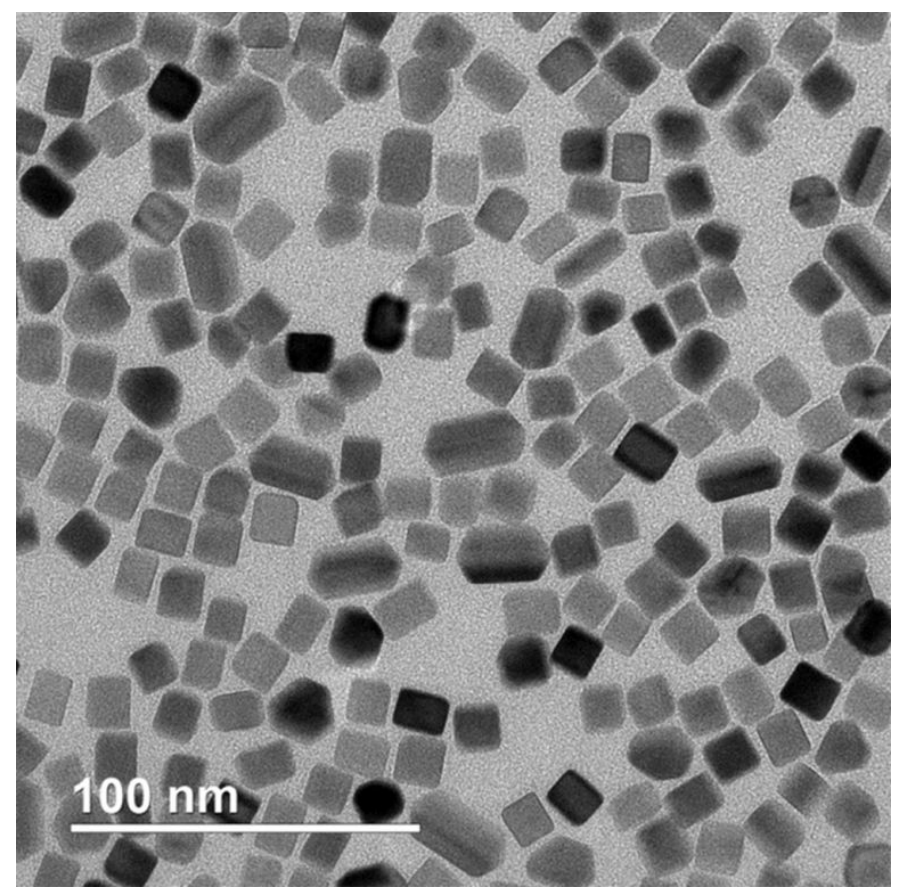

Figure S5. TEM image of Pd nanocrystals prepared using the standard protocol except that the reaction solution was bubbled with argon for 15 min to remove the dissolved oxygen prior to the nucleation step. 


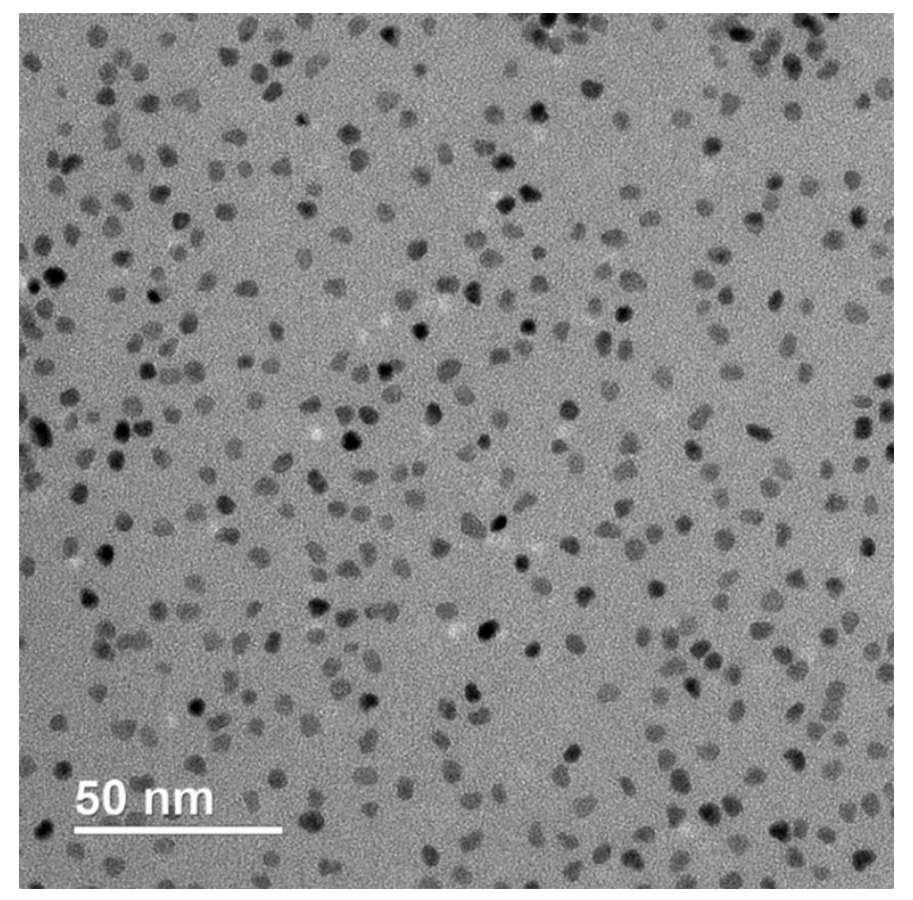

Figure S6. TEM image of Pt seeds obtained using a polyol synthesis that involves the reduction of a $\mathrm{Pt}(\mathrm{IV})$ precursor by EG in the presence of $\mathrm{KBr}$ and PVP. The nucleation was allowed to proceed in a tubular flow reactor at $185^{\circ} \mathrm{C}$ for $60 \mathrm{~s}$. 

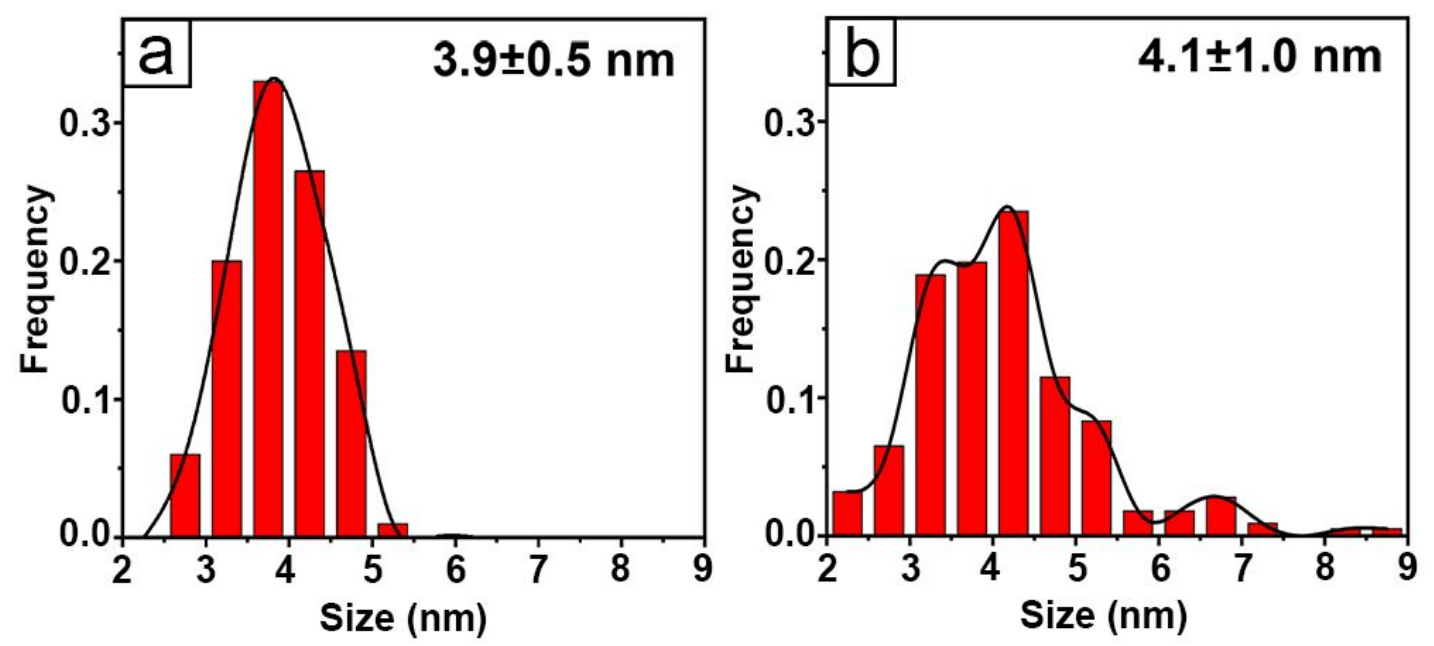

Figure S7. Histograms showing the size distribution of Pt nanocrystals obtained by (a) triggering the nucleation at $185^{\circ} \mathrm{C}$ for a duration of $60 \mathrm{~s}$, followed by growth at $110{ }^{\circ} \mathrm{C}$ for $48 \mathrm{~h}$ (i.e., the product shown in Figure 7a), and (b) holding the reaction solution at $185^{\circ} \mathrm{C}$ for $8 \mathrm{~h}$ (i.e., the product shown in Figure $7 \mathrm{~b}$ ), respectively. 